\title{
Review
}

Sven Schünke and Matthias Stoldt*

\section{Structural snapshot of cyclic nucleotide binding domains from cyclic nucleotide-sensitive ion channels}

\begin{abstract}
Cyclic nucleotide-binding domains (CNBDs) that are present in various channel proteins play crucial roles in signal amplification cascades. Although atomic resolution structures of some of those CNBDs are available, the detailed mechanism by which they confer cyclic nucleotide-binding to the ion channel pore remains poorly understood. In this review, we describe structural insights about cyclic nucleotide-binding-induced conformational changes in CNBDs and their potential coupling with channel gating.
\end{abstract}

Keywords: cAMP; cGMP; cyclic nucleotide-gated (CNG); hyperpolarization-activated and cyclic nucleotide-gated (HCN); NMR spectroscopy; X-ray crystallography.

*Corresponding author: Matthias Stoldt, Institute of Complex Systems, Structural Biochemistry (ICS-6), Research Centre Jülich, D-52425 Jülich, Germany; and Institut für Physikalische Biologie, Heinrich-Heine-Universität, D-40225 Düsseldorf, Germany, e-mail:m.stoldt@fz-juelich.de

Sven Schünke: Institute of Complex Systems, Structural Biochemistry (ICS-6), Research Centre Jülich, D-52425 Jülich, Germany

\section{Introduction}

Ion channels that are activated upon binding of cyclic nucleotides play key roles in regulating the excitation profile of heart muscle cells, as well as visual and olfactory neurons. These channels belong to the superfamily of voltage-gated cation channels and are subdivided into cyclic nucleotide-gated (CNG) channels and hyperpolarization-activated and cyclic nucleotide-gated (HCN) channels (Kaupp and Seifert, 2001, 2002; Robinson and Siegelbaum, 2003; Craven and Zagotta, 2006; Cukkemane et al., 2011). Like CNG channels, HCN channels are composed of several subunit types. Four mammalian HCN subunit isoforms (HCN1-HCN4) are known, each with distinct tissue distributions and biophysical properties. Despite their different physiological properties, the structures of HCN and CNG channels are closely related. Both channels are tetrameric proteins whose subunits are arranged around a central pore (Figure 1). Each subunit is composed of six transmembrane segments (S1-S6), including the voltage-sensor region located in S4 (with positively charged residues every third position), and intracellular $\mathrm{N}$ - and $\mathrm{C}$-terminal regions. The ion channel pore is formed by transmembrane segments S5 and S6, with a pore loop region positioned between the two segments that does not completely traverse the membrane. Cyclic nucleotides bind to the cyclic nucleotide-binding domain (CNBD) located at the C-terminus of each subunit. These CNBDs have a conserved fold when compared with other cyclic nucleotide-binding proteins, such as protein kinases $\mathrm{G}$ and A (PKG and PKA; Vaandrager and de Jonge, 1996; Kim et al., 2005), the bacterial cAMP-regulated catabolite gene activator protein (CAP) (Zubay et al., 1970; Eron et al., 1971; McKay and Steitz, 1981), the exchange protein directly activated by cAMP (EPAC) (Rehmann et al., 2003), and KCNH channels (Brelidze et al., 2012; Marques-Carvalho et al., 2012).

In HCN and CNG channels, the CNBD ( $\sim 120$ residues) is directly connected by a C-terminal linker (C-linker) to the transmembrane pore-segment S6 (Craven and Zagotta, 2006). The C-linkers of mammalian CNG and HCN channels consist of approximately 80 residues and are proposed to transfer ligand-binding to the channel gate (for a review see Craven and Zagotta, 2006; Cukkemane et al., 2011). HCN channels open on membrane hyperpolarization, and cyclic nucleotide binding modulates the activity of HCN channels by shifting the voltage dependence of activation to more positive potentials. In contrast, direct binding of cyclic nucleotides is required to open CNG channels, which are only driven weakly by depolarization. In CNG and HCN channels ligand-binding to the CNBD favors channel opening by conformational 


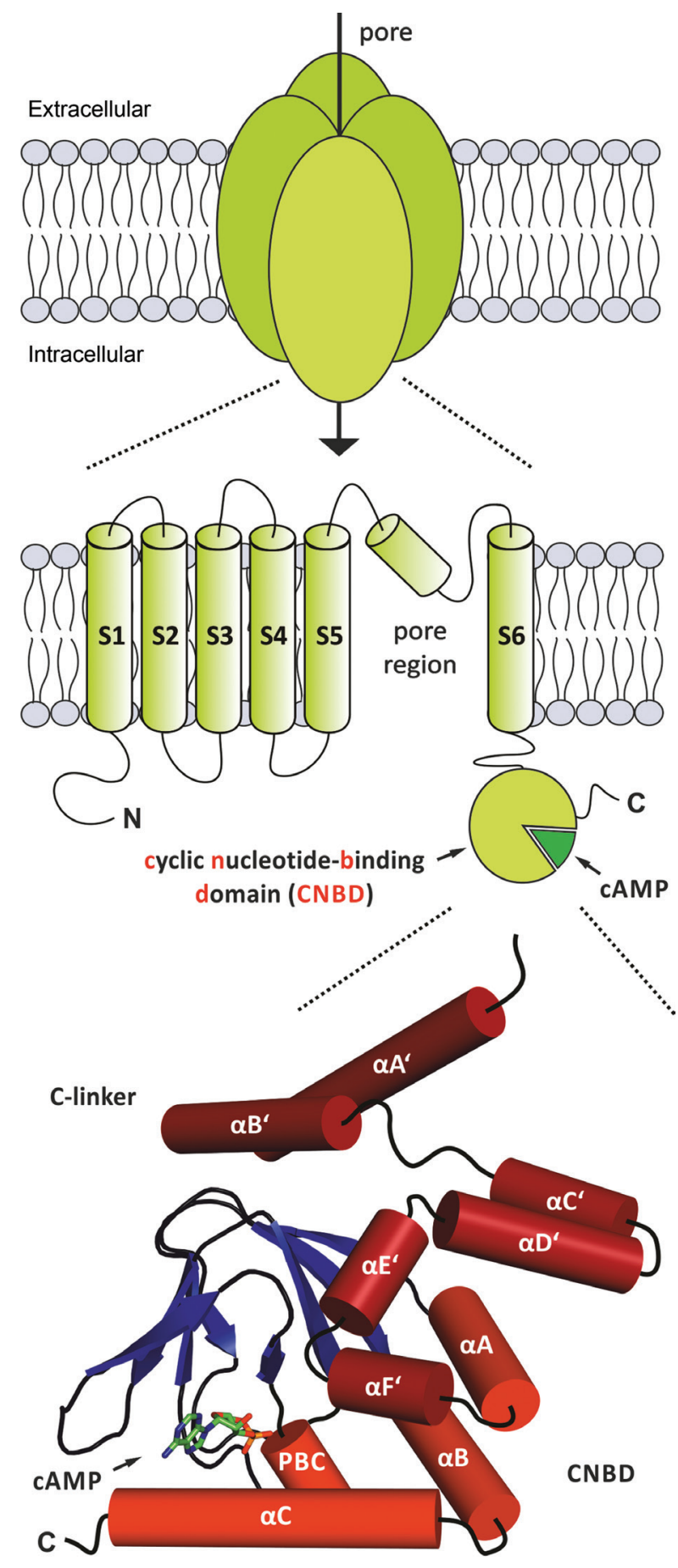

Figure 1 Topology of cyclic nucleotide-modulated ion channels: CNG and HCN channels are composed of four subunits (top).One subunit consists of six transmembrane segments (S1-S6) including an amino- and carboxy-terminal region (middle). The C-terminal region contains a CNBD. The $\mathrm{C}$-linker region of each subunit is composed of six $\alpha$ helices (helices $\alpha A^{\prime}-\alpha F^{\prime}$, in dark red) that directly connect the CNBD to transmembrane segment 66 (bottom). CNBDs consist of four $\alpha$-helices ( $\alpha A-\alpha C$ and a short phosphate-bindingcassette (PBC) helix, in light red) and a $\beta$ roll (in blue). The cyclic nucleotide-binding pocket is formed by parts of the $\beta$ roll, the PBC helix and helix $\alpha \mathrm{C}$. The cAMP molecule is shown as a stick model. changes in the CNBD that are likely to be propagated via the connecting C-linker to the channel pore. However, the underlying detailed mechanisms to activate these channels remain unresolved.

In the past 10 years, significant advances have been made in elucidating the structures of cyclic nucleotidesensitive ion channel CNBDs. A detailed structurefunction analysis of CNG and HCN channel CNBDs was initiated (see information given in Table S1) when the first CNBD crystal structures of the eukaryotic HCN2 and prokaryotic MloK1 channel were reported (Zagotta et al., 2003; Clayton et al., 2004).

Currently, neither a complete structure of HCN or CNG channels nor the conformational changes that occur upon ligand-binding and channel opening are known in detail. Addressing the question whether the CNBD of eukaryotic $\mathrm{HCN}$ or CNG channels undergo structural rearrangements upon ligand-binding in a manner that differs to the bacterial MloK1 CNBD (Clayton et al., 2004; Altieri et al., 2008; Schünke et al., 2009; Mari et al., 2011; Schünke et al., 2011) is not possible, because only structural information of one ligand-free eukaryotic CNBD is available (Taraska et al., 2009) in contrast to multiple structures in the ligandbound state (see Table S1).

\section{Structure of the HCN2 CNBD in the ligand-bound state}

First structural insights into cyclic nucleotide-dependent HCN channel modulation have been provided in 2003 by William Zagotta and co-workers. Here, the structure of the C-terminal fragment of the murine HCN2 channel was solved by X-ray crystallography and consisted of the CNBD and the C-linker region (mHCN2 CNBD) in the presence of cAMP or cGMP (Zagotta et al., 2003). The protein core of the mHCN2 CNBD is characterized by a $\beta$ roll, consisting of eight antiparallel aligned $\beta$-strands, topped by a helical bundle of four $\alpha$-helices (Figure 2). The $\beta$ roll is surrounded by the $\mathrm{N}$-terminal $\alpha$-helix $\mathrm{A}$, a short internal $\alpha$-helix located between $\beta$-strands 6 and 7, designated as the phosphate-binding-cassette (PBC), and two $\alpha$-helices $\mathrm{B}$ and $\mathrm{C}(\alpha \mathrm{B}$ and $\alpha \mathrm{C})$ located at the $\mathrm{C}$-terminus. This structure, together with previously determined structures of PKA, CAP and Epac, confirmed the $\beta$ roll topology to be conserved in all CNBDs (Weber and Steitz, 1987; Su et al., 1995; Diller et al., 2001; Rehmann et al., 2003).

The cyclic nucleotide binds inside a cavity formed by parts of the $\beta$ roll, the PBC helix and helix $\alpha \mathrm{C}$. The cAMP molecule is bound in the anti conformation, such 


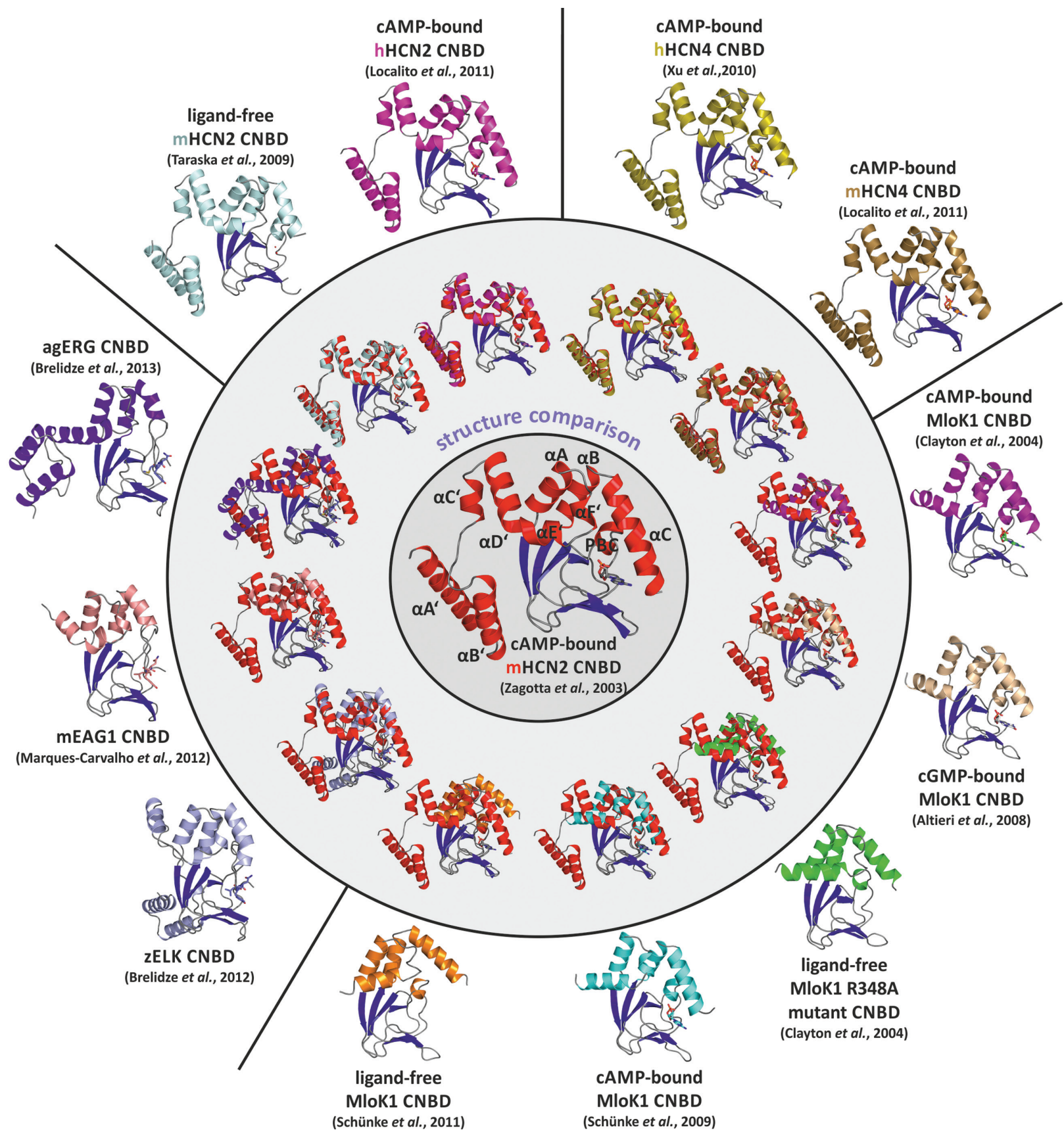

Figure 2 Structure overlay and comparison of CNBD structures from cyclic nucleotide-sensitive ion channels. Ribbon representations of cAMP-bound and -free mHCN2 CNBD (PDB ID codes: $1 Q 50$ and 3FFQ), cAMP-bound hHCN2 CNBD (PDB ID code: 3U10), cAMP-bound hHCN4 and mHCN4 CNBD (PDB ID codes: 30TF and 3U11), cAMP-bound, cGMP-bound wild-type and ligand-free R348A mutant MloK1 CNBD crystal structures (PDB ID codes: 1VP6, 3CL1, and 1U12), wild-type cAMP-bound and -free MloK1 CNBD solution structures (PDB ID codes: 2K0G and $2 \mathrm{KXL}$ ), zebrafish ELK, mouse EAG1 and Anopheles gambiae ERG (agERG) CNBD crystal structures in the 'self-liganded' state (PDB ID codes: 3UKN, 4F8A, and 4L11).

that the phosphoribose moiety interacts with parts of the $\beta$ roll and the PBC helix through electrostatic and hydrogen-bond interactions (Figure 3). One key residue, R591 of HCN2 located between the PBC helix and $\beta 7$, interacts directly with the negatively charged exocylic oxygen of the phosphate group. This arginine residue together with its interaction is conserved (Figure 4) and mutation of this residue in CNG, HCN and MloK1 channels decrease the affinity for cyclic nucleotides (Tibbs et al., 1998; Chen et al., 2001; Clayton et al., 2004; Cukkemane 
A
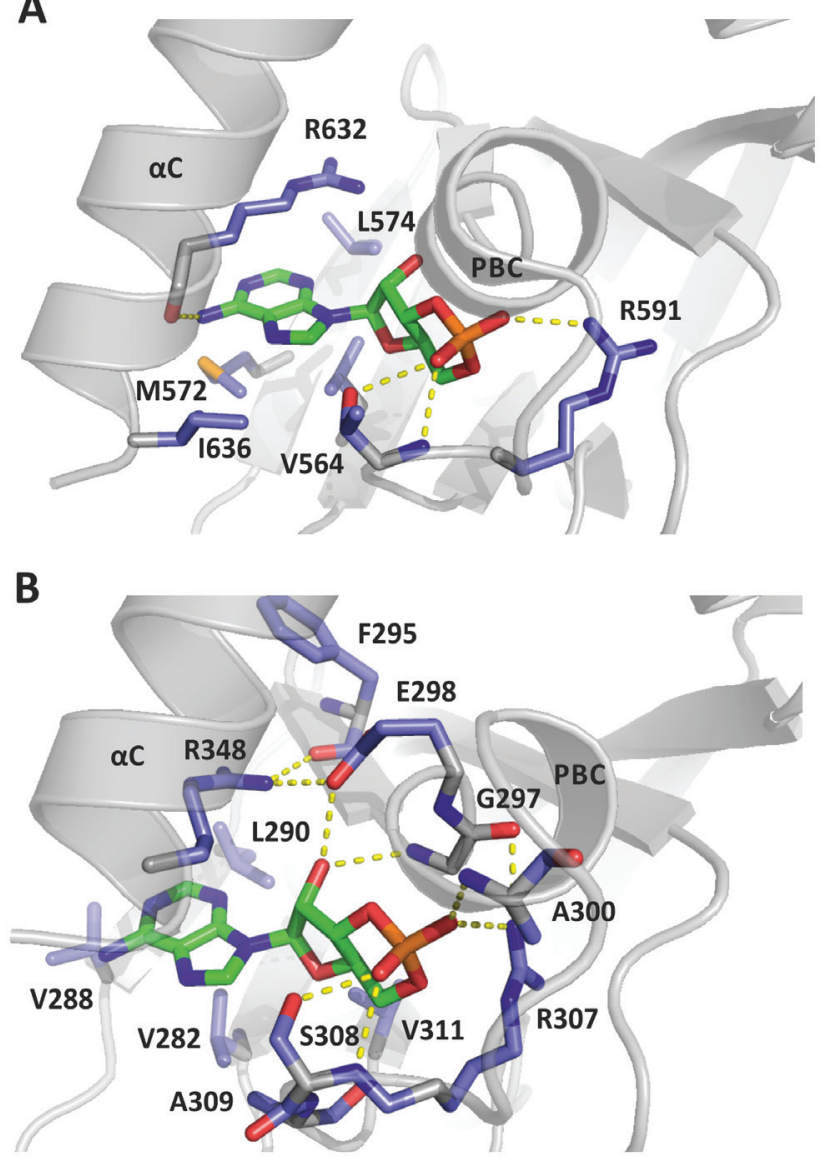

C

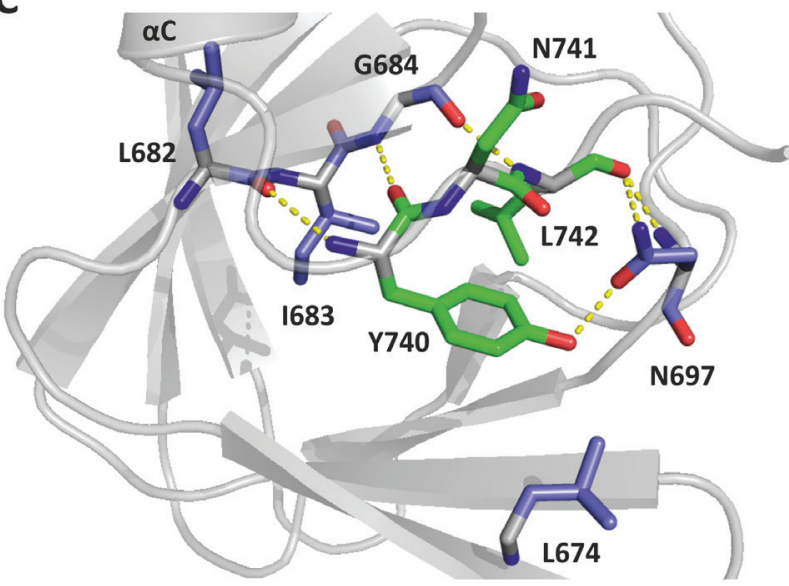

Figure 3 Cyclic nucleotide binding pockets of (A) mHCN2 (PDB ID code: 1Q50; Zagotta et al., 2003), (B) MloK1 (PDB ID code: 2K0G; Schünke et al., 2009), and (C) zELK (PDB ID code: 3UKN; Brelidze et al., 2012) CNBDs.Amino acid side chains that partially fill the binding pocket in the zELK structure are shown in green.

et al., 2007; Altieri et al., 2008; Harzheim et al., 2008; Schünke et al., 2009). The purine ring interacts through stacking and hydrogen-bond interactions with additional residues located in both the $\beta$ roll and helix $\alpha \mathrm{C}$. Van der Waals interactions with the purine ring of cAMP are formed by residue I636. Additionally, a hydrogen-bond to the N6 amine is formed by the backbone carbonyl oxygen of residue R632. This key residue located in helix $\alpha \mathrm{C}$ was later reported to control the effectiveness by which cAMP enhances channel opening (Zhou and Siegelbaum, 2007). Several additional van der Waals interactions are formed by residues R632 of helix $\alpha \mathrm{C}$, and V564, M572 and L574 located in the $\beta$ roll (Figure 3A). The interactions of helix $\alpha \mathrm{C}$ are of particular interest, because mutations of residues located in $\alpha \mathrm{C}$ in $\mathrm{HCN}$ and CNG channels impair ligand sensitivity and perturb channel function (Varnum et al., 1995; Matulef et al., 1999; Wainger et al., 2001).

Interestingly, in cGMP-bound structures the cGMP molecule exists in the syn configuration, thereby facilitating a hydrogen-bond interaction of the purine ring N2 atom with residue T592 located in the $\beta$ roll (Flynn et al., 2007). Several residues in helix $\alpha C$ (R632, R635, I636 and K638) are reported to increase selectivity of HCN2 channels for cAMP rather than for cGMP (Flynn et al., 2007; Zhou and Siegelbaum, 2007), however the reason for this selectivity is poorly understood.

\section{C-linker region of the HCN2 CNBD}

The first structural information about the C-linker (approximately 80 residues) that connects the CNBD with the transmembrane segment S6 was reported by crystallization of the HCN2 CNBD (Zagotta et al., 2003). In the crystal structure, the four C-linker subunits including the CNBD are assembled as tetramers with a four-fold axis of rotational symmetry, which is consistent with the tetrameric channel architecture. So far, no structural information is available for the C-linker in CNG channels. However, the $\mathrm{C}$-linker and CNBD structure from an invertebrate HCN channel designated SpIH, which shares sequence homology with CNG and HCN channels (Figure 4), shows a similar fold to that of HCN channels, suggesting that the C-linker of CNG channels exhibits a similar fold (Flynn et al., 2007). The C-linker is important in CNG and HCN channels, because it links the CNBDs to the ion channel gating machinery, and the majority of all intersubunit interactions of the four subunits in the crystal structure are provided between the $\mathrm{C}$-linker subunits without interactions between the CNBDs. The C-linker is composed of six $\alpha$-helices $\left(\alpha \mathrm{A}^{\prime}\right.$ to $\left.\alpha \mathrm{F}^{\prime}\right)$ and the first two helices of each subunit $\left(\alpha \mathrm{A}^{\prime}\right.$ and $\left.\alpha \mathrm{B}^{\prime}\right)$ form an antiparallel 'helix-turnhelix' motif that interacts with helix $\alpha \mathrm{C}^{\prime}$ and $\alpha \mathrm{D}^{\prime}$ of the neighboring subunit, i.e., like an 'elbow' resting on the 'shoulder' of its neighbor (Zagotta et al., 2003; Figure 


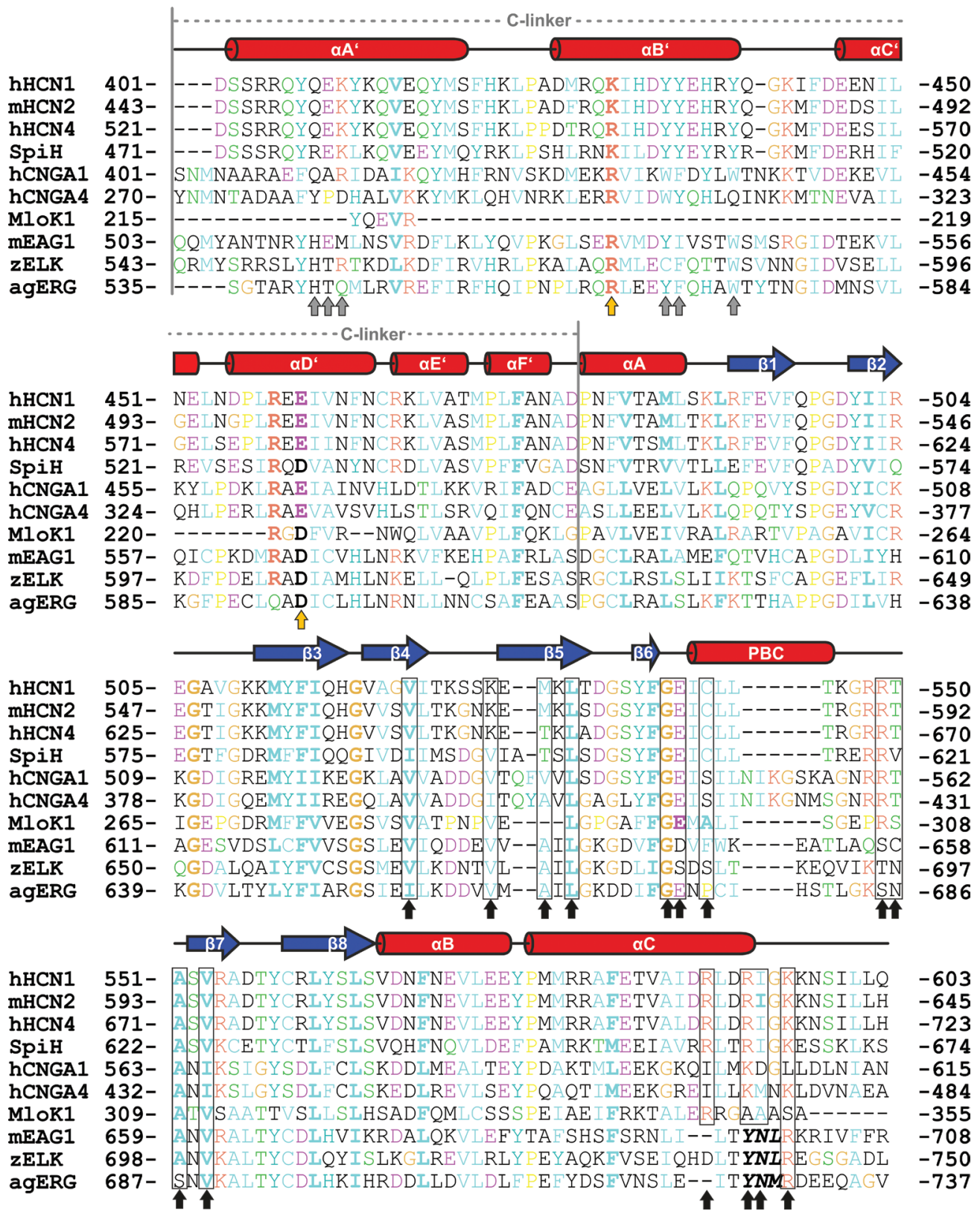

Figure 4 Sequence alignment of the CNBD and C-linker region from human HCN1 (hHCN1), mouse HCN2 (mHCN2), human HCN4 (hHCN4), the CNBD and C-linker of SpIH of Strongylocentrotus purpuratus (purple sea urchin), human CNGA1 (hCNGA1) and CNGA4 (hCNGA4), the CNBD of Mesorhizobium loti (MloK1), the CNBD and C-linker of mouse EAG1, zebrafish ELK (zELK) and Anopheles gambiae ERG (agERG).Secondary structure elements for the C-linker and CNBD of $\mathrm{mHCN} 2$ are shown (blue arrows indicate $\beta$ strands and red cylinders show $\alpha$ helical conformation). Residues involved in binding of cyclic nucleotides are highlighted by black arrows. Residues involved in C-linker intersubunit contacts are highlighted by grey arrows, the salt bridge between residues $\mathrm{K} 472$ in helix $\alpha \mathrm{B}^{\prime}$ and $\mathrm{E} 502$ in $\alpha \mathrm{D}^{\prime}$ of the neighboring subunit is highlighted by orange arrows. Residues involved in C-linker intersubunit contacts are highlighted by grey arrows. Residues involved in binding of cyclic nucleotides are framed and highlighted by black and grey arrows. Conserved residues are highlighted in bold letters and residues acting as an 'intrinsic ligand' for the mEAG1, zELK and agERG CNBD are marked in bold black italic.

5A). This conformation, designated as the 'gating ring' (Johnson and Zagotta, 2005), was first presented for HCN2 and later by structures of different HCN isoforms (Zagotta et al., 2003; Flynn et al., 2007; Craven et al., 2008; Taraska et al., 2009; Lolicato et al., 2011; Xu et al., 2010, 2012).
The C-linker subunits contact each other via hydrophobic interactions between the 'elbow-on-shoulder' motif, hydrogen bonds and a salt bridge between residues K472 in helix $\alpha \mathrm{B}^{\prime}$ and $\mathrm{E} 502$ in $\alpha \mathrm{D}^{\prime}$ of the neighboring subunit (Figure 4). Binding of cAMP to the CNBD promotes the 

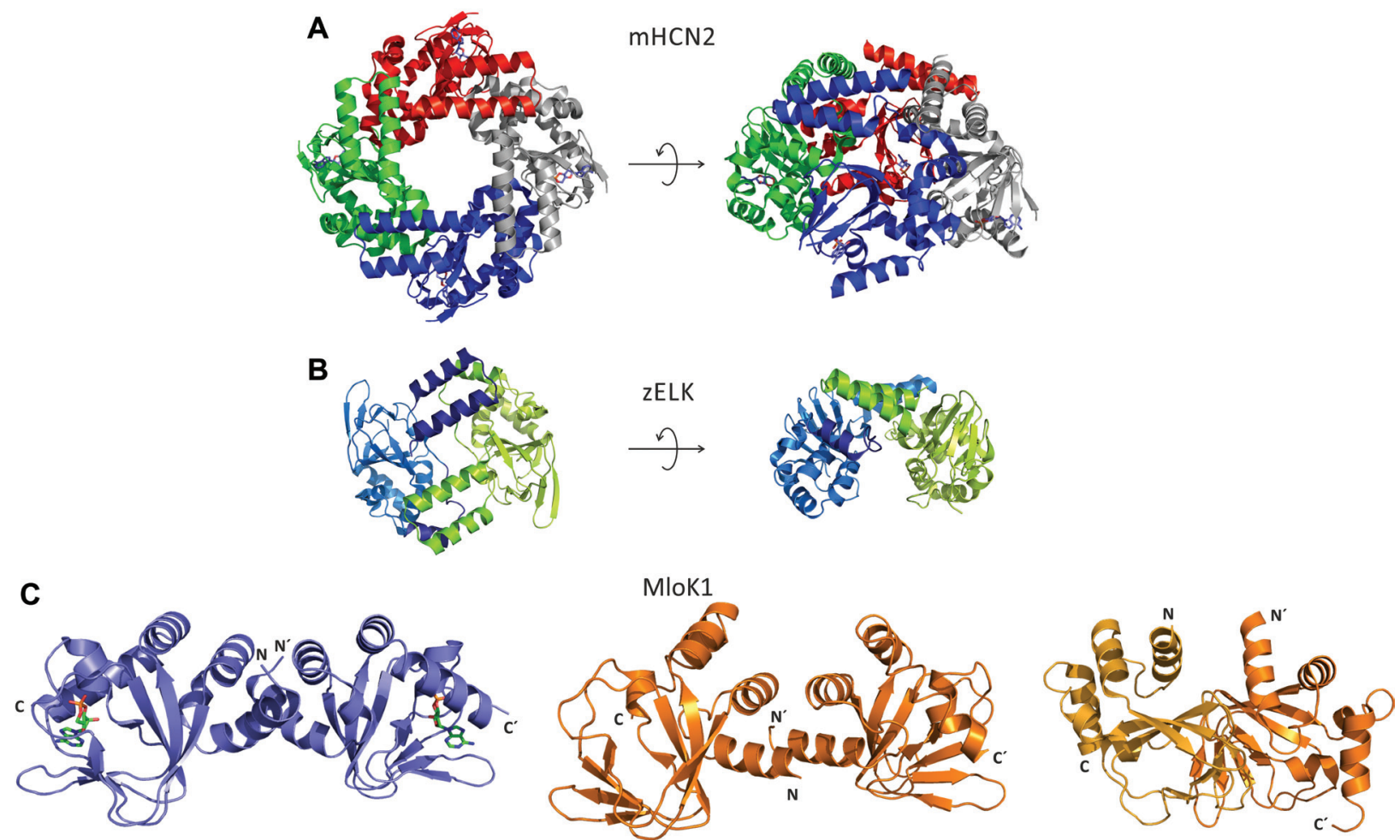

Figure 5 Multimerization and dimerization of CNBDs found in crystal structures.(A) mHCN2 (PDB ID code: 1Q50; Zagotta et al., 2003), (B) zELK (PDB ID code: 3UKN; Brelidze et al., 2012), (C) MloK1 [PDB ID codes: left (holo-state) 1VP6, center (apo-state) 1U12]; Clayton et al., 2004, right (apo-state, alternative CNBD dimerization interface) $3 \mathrm{CO}_{2}$; Altieri et al., 2008).

tetrameric assembly of these domains as shown by equilibrium sedimentation experiments of the CNBD with the C-linker (Zagotta et al., 2003; Lolicato et al., 2011). Thus, the C-linkers appear to play a crucial role in allosteric modulation of channel gating and show high sequence similarity among each other (Figure 4). Removal of the intersubunit salt bridge by mutagenesis in CNGA1 and HCN supports channel opening (Craven and Zagotta, 2004). Furthermore, the exchange of three consecutive

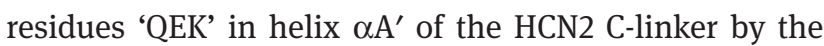
sequence 'FPN' present in CNGA4 channels promotes the disassembly of the tetrameric gating ring and cAMP behaves in a reverse manner that inhibits rather than activates the HCN2 channel (Zhou et al., 2004).

\section{Structure of HCN2 CNBD in the ligand-free state}

The only structural information of a CNBD from HCN channels without bound cyclic nucleotide was reported by the crystallization of the mHCN2 CNBD with the
C-linker (Taraska et al., 2009). Surprisingly, a comparison of the apo-state mHCN2 CNBD structure with the previously reported cAMP-bound state structure (Zagotta et al., 2003) showed that the conformations are near identical with an RMSD of 0.9 A (Figure 2). Minor structural differences include the overall $\alpha$-helical content caused by the absence of helix $\alpha \mathrm{F}^{\prime}$ and the shortening of helix $\alpha \mathrm{C}$ (by five residues) in the apo-state. However, crystal packing interactions in the region of helix $\alpha \mathrm{C}$ or putative ligand replacement by bromide ions located in the binding pocket may have forced the conformation of helix $\alpha \mathrm{C}$ in the apo-state structure, and therefore this structure may not represent the actual apo-state conformation in solution (Taraska et al., 2009; Puljung and Zagotta, 2013). In addition to these initial structural insights about possible conformational changes of the mHCN2 CNBD upon ligandbinding, extensive structural analysis with transition metal ion FRET experiments support the 'coil-to-helix' transition of helix $\alpha \mathrm{F}^{\prime}$ and a change in the length of helix $\alpha \mathrm{C}$ upon ligand-binding (Taraska et al., 2009; Puljung and Zagotta, 2013). Furthermore, in intact CNGA1 channels, homologous to HCN channels, it was shown that stabilization of helix $\alpha \mathrm{C}$ promoted channel opening by metal 
coordination by a pair of histidine residues (Puljung and Zagotta, 2013). In the same study, transition metal ion FRET results suggest movement of the $\mathrm{N}$-terminal region of helix $\alpha C$ closer to the $\beta$ roll core of the CNBD upon cyclic nucleotide binding. The $\mathrm{N}$-terminal region of helix $\alpha \mathrm{C}$ is in close proximity to helix $\mathrm{F}^{\prime}$ and it seems likely that the suggested movement of helix $\alpha \mathrm{C}$ could be further propagated via helix $\mathrm{F}^{\prime}$ to the $\mathrm{C}$-linker. Such a conformational rearrangement beginning from the $\mathrm{CNBD}$ via the $\mathrm{C}$-linker through the pore region could represent the initial event for stabilization of the open state of the channel pore.

\section{Model for ligand-induced conformational changes of CNBDs in HCN channels}

Based on first models (Craven and Zagotta, 2006; Rehmann et al., 2007) and their new data, Zagotta and co-workers proposed a recent model for ligand-induced conformational changes of the CNBD in HCN channels (Taraska et al., 2009; Puljung and Zagotta, 2013):

i. In the absence of cyclic nucleotides the channel pore is closed (unbound/closed state) and the $\alpha \mathrm{C}$ region adopts a more flexible conformation rather than a stable $\alpha$-helix.

ii. Upon ligand-binding to the pocket formed by the PBC helix and regions of the $\beta$ roll, the ion channel pore remains closed (bound/closed state).

iii. Next, helix $\alpha \mathrm{C}$ and $\mathrm{F}^{\prime}$ adopt a helical conformation that moves helix $\alpha \mathrm{C}$ like a 'lid' above the ligandbinding site. Ordering and movement of helix $\alpha \mathrm{C}$ is finally coupled through the C-linker to open the channel pore (bound/open state).

\section{Structure of the HCN4 channel CNBD in the ligand-bound state}

The solved crystal structure of the mHCN2 CNBD including the $\mathrm{C}$-linker has provided the structural basis for cyclic nucleotide-dependent HCN channel gating. However, there was a paucity of structural information of CNBDs from other HCN channel isoforms. To address this point, $\mathrm{Xu}$ et al. solved the crystal structure of the cAMP-bound human HCN4 CNBD, including the C-linker (hHCN4 CNBD) (Xu et al., 2010). The overall structure of the hHCN4 CNBD and subsequently the mouse HCN4 CNBD (Lolicato et al., 2011) are very similar to the previously determined mHCN2 CNBD structure (Figure 2) and showed a four-fold symmetry arrangement of the protein with intersubunit interactions predominantly formed by the C-linkers from neighboring subunits. Functional characterization through electrophysiological experiments of a chimeric HCN channel consisting of the hHCN4 CNBD and C-linker connected to the mHCN2 transmembrane part (Figure 4) showed a three-fold reduction in response to cAMP when compared to the full-length mHCN2 channel (Xu et al., 2010). Based on marginal differences in the loop region between the strands $\beta 4$ and $\beta 5$ in the hHCN4 CNBD structure compared with the mHCN2 CNBD structure, the authors suggest that residue T650 in hHCN4 and M572 in mHCN2 close to this loop region (Figure 4) could partially contribute to the observed differences in the cAMPresponse. The point mutation M572T in mHCN2 reduced the response to cAMP. It is likely that a hydrophobic residue such as methionine is probably preferred over a hydrophilic residue such as threonine. However, the converse T650M point mutation, which represents the equivalent position to interact with the purine base moiety in the hHCN4 channel, did not reveal an increase in response to cAMP.

\section{Structure of the HCN1 channel CNBD in the ligand-bound state}

The three most characterized HCN channel isoforms HCN1, HCN2 and HCN4 exhibit different responses to cyclic nucleotide-binding. HCN2 and HCN4 channels require a saturating amount of cAMP to shift the activation curve by $+17 \mathrm{mV}$, whereas HCN1 shifts the activation curve considerably less in response to increasing cAMP levels, with values of $+4 \mathrm{mV}$ (Chen et al., 2001). Surprisingly, cAMP dose-response curves for HCN1 and HCN2 obtained from inside-out patch clamp recordings showed similar nanomolar affinities for the activation of both channels, suggesting that the minor shift of the HCN1 activation curve is not related to a lower binding affinity of the HCN1 isoform for cAMP (Chen et al., 2001; Wang et al., 2001). Interestingly, a combined approach of structural, biochemical and biophysical characterization of the HCN1, HCN2, and HCN4 CNBDs including the C-linker regions revealed that the HCN1 construct tetramerizes when half-saturated with cAMP (Lolicato et al., 2011), and this observation contrasts to the HCN2 and HCN4 isoforms that require saturating amounts of cAMP (Zagotta et al., 2003; Xu et al., 2010). This would explain why HCN1 shows 
a weaker response to cAMP in contrast to the HCN2 and HCN4 isoforms, because the HCN1 CNBD is already partly present in a tetrameric state under non-saturating ligand conditions. This observation was confirmed by voltage clamp experiments, which showed that a shifted position to a more positive value of the half-activation voltage $\left(\mathrm{V}_{1 / 2}\right)$ in HCN1 correlates with its behavior to be present as a tetramer in vitro (Lolicato et al., 2011).

Lolicato et al. solved the crystal structures of the C-linker region and CNBD from the mouse HCN1, human HCN2, and mouse HCN4 channel all in the presence of cAMP. The amino acid sequence of all these HCN CNBDs (including the C-linker) from mouse and human are completely identical except for one point mutation $\mathrm{S}\left({ }_{\mathrm{m}} 440 / \mathrm{h} 451\right) \mathrm{N}$ in the HCN1 CNBD. This study provided new structural information about the HCN1 CNBD.

The holo-state mouse HCN1 CNBD (mHCN1 CNBD) plus the $\mathrm{C}$-linker was found to crystallize with a four-fold symmetry, as shown previously for mHCN2 and hHCN4 (Zagotta et al., 2003; Xu et al., 2010). The structure of the mHCN1 CNBD in complex with cAMP is virtually identical to the structures of the ligand-bound HCN2 and HCN4 CNBDs plus the $\mathrm{C}$-linker domain.

An overlay of these structures together with all previously determined holo-state HCN CNBD structures of different isoforms results in an average root mean square deviation of around $1 \AA$ A. Minor differences are only located in the loop region between $\beta 4$ and $\beta 5$ strands, positioned below the base ring of the cyclic nucleotide, where most differences in the primary sequence between the three HCN isoforms are clustered. It was suggested previously that this region could partially contribute to different cAMP-responses of different HCN isoforms (Xu et al., 2010).

\section{Structural basis of the MloK1 CNBD in the ligand-free and -bound states}

The prokaryotic $\mathrm{K}^{+}$-selective $\mathrm{CNG}$ channel MloK1 has been identified in the bacterium Mesorhizobium loti (Clayton et al., 2004; Nimigean et al., 2004) and shares a similar topology with eukaryotic CNG and HCN channels (Figure 1). The channel consists of four identical subunits, each exhibiting six transmembrane segments (S1-S6), a 'GYG' signature sequence for $\mathrm{K}^{+}$selectivity and a conserved CNBD. The length of the C-linker region in MloK1 that connects the CNBD to S6 is noticeably shorter ( $\sim 20$ residues) when compared with the mammalian CNG and HCN channels ( $\sim 80$ residues). Crystal structures of the mutant and wild-type CNBD in the ligand-free and -bound state, respectively, revealed insights into the conformational rearrangements in the CNBD upon ligand-binding (Clayton et al., 2004; Altieri et al., 2008). The structure of the MloK1 CNBD shows the characteristic properties similar to the HCN2 CNBD structure (Figures 1 and 2), consisting of a $\beta$ roll core topped by a bundle of four $\alpha$-helices and a short PBC helix (Zagotta et al., 2003). Thus, the overall structure is highly conserved in all CNBDs (Weber and Steitz, 1987; Su et al., 1995; Diller et al., 2001; Rehmann et al., 2003).

The cyclic nucleotide is bound inside a cavity of the MloK1 CNBD formed by parts of the $\beta$ roll, the PBC helix and helix $\alpha \mathrm{C}$. The conserved residues R307, A300 and S308 (Figure 4) undergo electrostatic and hydrogen-bonding interactions with the exocyclic oxygen of the phosphate group (Figure 3B). Residue G297 and the carboxy-group of E298 exert additional hydrogen-bonding interactions with the 2'-hydroxy-group of the ribofuranose. Several hydrophobic residues (V282, V288, L290, A309 and V311) are directly located below the purine base and form strong van der Waals contacts with the purine base. The side chain of the key residue R348 reaches over the purine base, forms polar contacts with residues F295 and E298 and additionally acts as a lid on the binding cavity. The exchange of one of the respective arginine residues (R307 or R348) impaired cyclic nucleotide-sensitivity (Clayton et al., 2004; Cukkemane et al., 2007; Altieri et al., 2008).

In the crystal structures, the CNBDs of MloK1 are arranged as dimers and the dimer interface is formed by the short C-linker, which has been proposed to be involved in channel gating (Figure 5C). However, a cryo-electron microscopy structure of the full-length MloK1 channel in the presence of cAMP at $16 \AA$ resolution revealed a fourfold symmetric arrangement of the CNBDs separated by discrete gaps (Chiu et al., 2007). Ligand-binding studies showed that the whole channel and the monomeric CNBD bind cAMP non-cooperatively with similar affinity (Cukkemane et al., 2007). Taken together these structural and biochemical studies suggest that the ligand-binding events of each subunit are independent from each other. The observation that the CNBD exists as a monomeric protein in solution was further supported by structural characterization of the MloK1 CNBD by NMR spectroscopy (Schünke et al., 2007; Schünke et al., 2009). The solution structure clearly shows a monomeric fold and the $\mathrm{N}$-terminal helix $\alpha \mathrm{A}^{\prime}$ is straight in the solution structure rather than bent as observed in the crystal (Figure 2). This observation suggests that the predominant dimeric arrangement in the crystal is not a physiologically relevant conformation in the prokaryotic MloK1 CNG compared to classical CNG channels. 
Interestingly, an alternative CNBD dimerization interface was reported (Gushchin et al., 2012), which is present in the MloK1 CNBD point mutant R307W homodimer (Altieri et al., 2008, Figure 5C) and the Epac2 heterodimer (Rehmann et al., 2003, 2006) crystal structures. In contrast to the reported predominant MloK1 CNBD dimer interface present in the crystal structure (Clayton et al., 2004), molecular dynamics simulations showed that the novel interface is stable and no structural rearrangements were observed. A comparison with CNBD crystal structures from both proteins in the ligand-bound state (Clayton et al., 2004; Rehmann et al., 2008) shows that the conformational change of the CNBD upon ligand-binding contradicts this novel interface, suggesting that the dissociation of CNBD dimers upon ligand-binding could be an alternative mechanism of ligand gating in the MloK1 channel. This observation is consistent with the previously reported low resolution electron microscopy structure of the MloK1 channel in the presence of cAMP, where the CNBDs are completely separated by discrete spacing (Chiu et al., 2007).

Crystal structures of the mutant MloK1 CNBD in the apo-state revealed that the relative position of the $\mathrm{C}$-terminal helix $\alpha \mathrm{C}$ in the helical portion is not precisely defined. Helix $\alpha \mathrm{C}$ is disordered or partially unfolded and in some structures no electron density could be observed for this particular region (Clayton et al., 2004; Altieri et al., 2008). However, the solution structure of the wild-type MloK1 CNBD in the apo-state (Figure 2) shows that the position of helix $\alpha \mathrm{C}$ is well defined and adopts a single orientation (Schünke et al., 2011). Moreover, comparison of the wild-type cAMP-free and -bound CNBD solution structures shows large conformational rearrangements of the complete helical bundle on ligand-binding. Here, helix $\alpha B$ and $\alpha \mathrm{C}$ move closer to the ligand-binding cavity, and this displaces $\alpha \mathrm{A}$ relative to $\alpha \mathrm{A}^{\prime}$, and a sliding movement of the $\mathrm{N}$-terminal helix $\alpha \mathrm{A}^{\prime}$ across the $\beta$ roll is observed.

\section{Structural characterization of the transmembrane region of the MloK1 channel}

The tetrameric organization of the channel was confirmed by a cryo-electron microscopy structure of the MloK1 channel (Chiu et al., 2007) and a crystal structure of the MloK1 transmembrane segments in the closed state with a resolution of $3.1 \AA$ (Clayton et al., 2008). However, the CNBDs were not resolved in the crystal structure.
Interestingly, the structure of the S1-S4 transmembrane segments revealed a more compact fold with various interactions between all transmembrane segments. This is very different when compared with the classical voltage-sensor domain of voltage-gated ion channels. Furthermore, the MloK1 structure supports the already established suggestion that the main function of the S1-S4 segments, including the associated linker, is probably not to act as a voltage-sensor. The authors assumed that the S1-S4 segments could promote channel gating in two possible ways:

i. The region could restrict channel opening until an unknown stimulus directly affects this conformation.

ii. The region could work against CNBDs to enforce the pore closure in the ligand-free state. Furthermore, the center of the MloK1 channel cavity is occupied by phenylalanine side chains and conformational changes of CNBDs may affect the movement of the inner helices to regulate these side chains positions.

\section{Initial model for ligand-induced conformational changes of MloK1 CNBDs}

Further understanding about conformational changes in the MloK1 channel that occur upon ligand-binding and -dissociation was provided by an atomic force microscopy (AFM) study (Mari et al., 2011). Although ligand-induced conformational changes could not be observed for the wild-type MloK1 channel, the CNBD R348A mutant, which has a lower affinity for cAMP and thereby allows the preparation of the ligand-bound and -free state (Clayton et al., 2004; Cukkemane et al., 2007; Altieri et al., 2008; Peuker et al., 2013), was used to study conformational changes of the CNBDs upon ligand-binding and -dissociation. In the ligand-bound state, the CNBDs are arranged with a four-fold symmetry displayed by a cryo-electron microscopy structure (Chiu et al., 2007). The AFM topographs (Mari et al., 2011) showed that upon ligand-binding and dissociation a reversible conformational change of the mutant CNBDs occurs. In the ligand-bound state the fourfold symmetrically arranged conformation of the CNBDs is positioned close to the membrane surface. However, upon ligand dissociation the CNBD shows a noticeable expansion in height away from the transmembrane region (by approximately $1.7 \mathrm{~nm}$ ).

Cyclic nucleotide-binding studies of the full-length MloK1 channel and the isolated CNBD showed that the ligand-binding event differs between both constructs, 
suggesting an allosteric control of the CNBD by the transmembrane region of the MloK1 channel (Peuker et al., 2013). Moreover, two allosteric point mutations, K238A and C331L, located between helices $\alpha \mathrm{A}^{\prime}$-loop- $\alpha \mathrm{A} / \alpha \mathrm{C}$ and $\alpha \mathrm{B} / \alpha \mathrm{C}$ in the CNBD affected exclusively the dissociation rates, suggesting that binding of cyclic nucleotides follows an induced-fit mode.

\section{Structural basis of the zebrafish ELK channel cyclic nucleotide-homology domain (CNBHD)}

KCNH channels belong to the superfamily of voltage-gated potassium channels that also include the CNG and HCN channel families. These channels regulate repolarization of the action potential in the heart, neuronal excitation and proliferation of tumor cells. Unlike their cyclic nucleotide-regulated counterparts, $\mathrm{KCNH}$ channels are not regulated by direct binding of cyclic nucleotides. They have a similar channel topology as CNG and HCN channels and carry a Per-ARNT-Sim domain (PAS; first identified by sequence homology in the Drosophila proteins period and single-minded, and the vertebrate aryl hydrocarbon receptor nuclear transporter) at the N-terminus and a C-terminal region that carries a cyclic nucleotidebinding homology domain (CNBHD) and a C-linker that connects the CNBHD to the transmembrane segment S6. The family of KCNH channels can be subdivided into ether-à-go-go (EAG), EAG-related gene (ERG) and EAG-like (ELK) $\mathrm{K}^{+}$-channels.

The structure of the C-terminal region of a $\mathrm{KCNH}$ channel was solved for the zebrafish ELK (zELK) CNBHD, including the C-linker, at $2.2 \AA$ resolution (Brelidze et al., 2012). Overall the structure has a very similar fold to structures of the C-terminal regions from different HCN channels (Figure 2). However, the structure shows crucial differences in particular regions. Interestingly, the PBC helix is missing and most of the residues that are known to be crucial for interactions to cyclic nucleotides in CNBD structures of HCN channel isoforms, are not conserved in the zELK CNBHD. Additionally, residues in the region of the putative binding pocket induce a predominant negatively charged protein surface in the zELK CNBHD structure. This observation may explain why ELK is insensitive to cyclic nucleotide binding. Surprisingly, the putative cyclic nucleotide-binding cavity was filled by three residues (Y740 to L742; Figure 3C) that formed a short additional $\beta$ strand ( $\beta 9$ ) in the $\mathrm{C}$-terminal region after helix $\alpha$ C. Residues in $\beta 9$ are likely stabilized by various hydrogen-bond interactions with residues in the $\beta$ roll cavity of the zELK CNBHD. Residues Y740 and L742 in $\beta 9$ are oriented in analogous positions to the purine ring and the cyclic phosphate in CNBDs of HCN channels, respectively. Interestingly, mutations in $\beta 9$ shifted the voltage dependence of activation to larger depolarized voltages, suggesting that $\beta 9$ may act as an intrinsic ligand for ELK channels (Brelidze et al., 2012). Unexpectedly, the C-linker conformation in the zELK CNBHD structure is different to C-linker structures of HCN channel isoforms. In the crystal, the subunits form dimers instead of tetramers and helices $\alpha \mathrm{A}^{\prime}$ and of one subunit rest on helix $\alpha \mathrm{D}^{\prime}$ and the adjacent loop region towards helix $\alpha \mathrm{C}^{\prime}$ of the neighboring subunit (Figure 5B).

The structural basis of the zELK CNBHD including the C-linker region raises some questions: Is the intrinsic-ligand conformation a characteristic feature of KCNH CNBHDs? Does any unliganded conformation exist for KCNH CNBHDs? If so, provide the quaternary state of the CNBHD including the C-linker in full-length channels a two-fold or four-fold symmetry?

\section{Structure of the mouse EAG1 channel CNBHD}

A second CNBHD crystal structure of the mouse EAG1 channel was solved at $2.2 \AA$ resolution (Marques-Carvalho et al., 2012). There, unfortunately, the structural information for the mEAG1 C-linker region was not complete. A comparison of the mEAG1 CNBHD with the zELK CNBHD structure clearly shows strong similarities (Figure 2). The mEAG1 CNBHD structure shares many of the structural features present in the zELK CNBHD, including the self-liganded conformation. Residues Y699 and L701 of $\beta 9$ in the C-terminal region of the CNBHD are bound in similar positions compared to cyclic nucleotides in HCN CNBDs. Moreover, the mEAG1 structure supports the idea that the bound intrinsicligand conformation seems to be a conserved feature of all CNBHDs. Such an observation explains the absence of cyclic nucleotide binding to the mEAG1 CNBHD, as previously shown (Brelidze et al., 2009). Further biochemical characterization suggests that the mEAG1 CNBHD may exists in the unliganded conformation. Mutations that destabilize the self-liganded conformation should affect the voltage dependence of channel activation, indicating that the mEAG1 CNBHD may play a role in channel gating. 


\section{Structure of the mosquito ERG channel CNBHD}

Very recently, the structure of the mosquito ERG (agERG) channel CNBHD, including the C-linker, was determined at 2.5 ̊̊ resolution (Brelidze et al., 2013). Not surprisingly, the overall structure of the CNBHD shows the characteristic features of previously determined CNBHDs from mEAG and zELK channels (Figure 2), including the self-liganded conformation. The C-terminal residues $\mathrm{Y} 727$ to $\mathrm{M} 729$ of $\beta 9$ are bound in similar positions when compared with cyclic nucleotides in HCN CNBDs, explaining the lack of direct modulation of agERG channels by cyclic nucleotides (Brelidze et al., 2009, 2012, 2013).

Interestingly, the C-linker of the agERG CNBHD shows a different conformation in the crystal compared to previously reported CNBHD structures of ELK and HCN channels. In this study the agERG CNBHD crystallized as a monomer, whereas the zELK CNBHD and the HCN2 CNBD structures were solved as a dimer and tetramer, respectively. The C-linker of the agERG CNBHD consists of four $\alpha$-helices $\left(\alpha \mathrm{A}^{\prime}\right.$ to $\left.\alpha \mathrm{D}^{\prime}\right)$, that form two antiparallel 'helix-turn-helix' motifs with helices $\alpha \mathrm{A}^{\prime} / \alpha \mathrm{B}^{\prime}$ and $\alpha \mathrm{C}^{\prime} / \alpha \mathrm{D}^{\prime}$ ' showing the typical 'elbow-onthe-shoulder' arrangement, which is conserved in ELK and HCN structures. However, the intramolecular interface is formed by four $\alpha$-helices in the agERG channel, which contrasts the intermolecular formation by six $\alpha$-helices observed in the ELK and HCN channel structures. Although the ERG, ELK and HCN channels have high sequence similarities in the CNBHD/CNBD and $\mathrm{C}$-linker regions, the different $\mathrm{C}$-linker conformations observed in their crystal structures suggest that the C-linker is dynamic (Brelidze et al., 2013). It remains unclear whether the various conformations and the different oligomeric states of these channels observed in the crystals reflect different conformational states, channel type-dependencies, or result from the different constructs used.

\section{References}

Altieri, S.L., Clayton, G.M., Silverman, W.R., Olivares, A.O., De la Cruz, E.M., Thomas, L.R., and Morais-Cabral, J.H. (2008). Structural and energetic analysis of activation by a cyclic nucleotide binding domain. J. Mol. Biol. 381, 655-669.

Brelidze, T.I., Carlson, A.E., and Zagotta, W.N. (2009). Absence of direct cyclic nucleotide modulation of mEAG1 and hERG1

\section{Conclusion and outlook}

Structural information of many cyclic nucleotide-sensitive ion channel binding domains have been obtained and quite a large amount of biochemical, biophysical and physiological data on their functional properties has been collected during the last decade. However, the understanding of the structural biology of these proteins is still not complete. Despite these great advances, understanding the sequence of events occurring from ligand-binding to channel gating in both, CNG and HCN channels, remains to be elucidated.

Currently, neither complete structures of $\mathrm{HCN}$ or CNG channels nor conformational changes that occur upon ligand-binding and channel opening are known in much detail. The need to address the question, whether CNBDs of $\mathrm{HCN}$ or CNG channels undergo structural rearrangements upon ligand-binding similar to the bacterial MloK1 CNBD, becomes particularly clear with the fact that only a single eukaryotic CNBD crystal structure in the apo-state is available. The understanding of the allostery of these proteins, in particular resolving intersubunit interactions and conformational movements in a spatiotemporal precision, is a challenge for the future that will be investigated by combinational approaches using structural biology, biochemistry, functional genomics, and computational methods.

Acknowledgments: We thank Dieter Willbold and Andrew Dingley for stimulating discussions and careful reading of the manuscript. We gratefully acknowledge support (and training) from the International NRW Research School BioStruct, granted by the Ministry of Innovation, Science and Research of the State North Rhine-Westphalia, the Heinrich-Heine-Universität of Düsseldorf, and the Entrepreneur Foundation at the Heinrich-Heine-Universität of Düsseldorf.

Received July 26, 2013; accepted September 4, 2013; previously published online September 7, 2013 channels revealed with fluorescence and electrophysiological methods. J. Biol. Chem. 284, 27989-27997.

Brelidze, T.I., Carlson, A.E., Sankaran, B., and Zagotta, W.N. (2012). Structure of the carboxy-terminal region of a KCNH channel. Nature 481, 530-533.

Brelidze, T.I., Gianulis, E.C., Dimaio, F., Trudeau, M.C., and Zagotta, W.N. (2013). Structure of the C-terminal region of an 
ERG channel and functional implications. Proc. Natl. Acad. Sci. USA 110, 11648-11653.

Chen, S., Wang, J., and Siegelbaum, S.A. (2001). Properties of hyperpolarization-activated pacemaker current defined by coassembly of HCN1 and HCN2 subunits and basal modulation by cyclic nucleotide. J. Gen. Physiol. 117, 491-504.

Chiu, P.L., Pagel, M.D., Evans, J., Chou, H.T., Zeng, X., Gipson, B., Stahlberg, H., and Nimigean, C.M. (2007). The structure of the prokaryotic cyclic nucleotide-modulated potassium channel MloK1 at 16 A resolution. Structure 15, 1053-1064.

Clayton, G.M., Silverman, W.R., Heginbotham, L., and Morais-Cabral, J.H. (2004). Structural basis of ligand activation in a cyclic nucleotide regulated potassium channel. Cell 119, 615-627.

Clayton, G.M., Altieri, S., Heginbotham, L., Unger, V.M., and Morais-Cabral, J.H. (2008). Structure of the transmembrane regions of a bacterial cyclic nucleotide-regulated channel. Proc. Natl. Acad. Sci. USA 105, 1511-1515.

Craven, K.B. and Zagotta, W.N. (2004). Salt bridges and gating in the $\mathrm{COOH}$-terminal region of $\mathrm{HCN} 2$ and CNGA1 channels. J. Gen. Physiol. 124, 663-677.

Craven, K.B. and Zagotta, W.N. (2006). CNG and HCN channels: two peas, one pod. Ann. Rev. Physiol. 68, 375-401.

Craven, K.B., Olivier, N.B., and Zagotta, W.N. (2008). C-terminal movement during gating in cyclic nucleotide-modulated channels. J. Biol. Chem. 283, 14728-14738.

Cukkemane, A., Gruter, B., Novak, K., Gensch, T., Bonigk, W., Gerharz, T., Kaupp, U.B., and Seifert, R. (2007). Subunits act independently in a cyclic nucleotide-activated $\mathrm{K}^{+}$channel. EMBO Rep. 8, 749-755.

Cukkemane, A., Seifert, R., and Kaupp, U.B. (2011). Cooperative and uncooperative cyclic-nucleotide-gated ion channels. Trends Biochem. Sci. 36, 55-64.

Diller, T.C., Madhusudan, Xuong, N.H., and Taylor, S.S. (2001). Molecular basis for regulatory subunit diversity in CAMP-dependent protein kinase: crystal structure of the type II beta regulatory subunit. Structure 9, 73-82.

Eron, L., Arditti, R., Zubay, G., Connaway, S., and Beckwith, J.R. (1971). An adenosine $3^{\prime}: 5^{\prime}$-cyclic monophosphate-binding protein that acts on the transcription process. Proc. Natl. Acad. Sci. USA 68, 215-218.

Flynn, G.E., Black, K.D., Islas, L.D., Sankaran, B., and Zagotta, W.N. (2007). Structure and rearrangements in the carboxy-terminal region of SplH channels. Structure 15, 671-682.

Gushchin, I.Y., Gordeliy, V.I., and Grudinin, S. (2012). A novel dimerization interface of cyclic nucleotide binding domain, which is disrupted in presence of CAMP: implications for CNG channels gating. J. Mol. Model. 18, 4053-4060.

Harzheim, D., Pfeiffer, K.H., Fabritz, L., Kremmer, E., Buch, T., Waisman, A., Kirchhof, P., Kaupp, U.B., and Seifert, R. (2008). Cardiac pacemaker function of HCN4 channels in mice is confined to embryonic development and requires cyclic AMP. Embo J. 27, 692-703.

Johnson, J.P. Jr. and Zagotta, W.N. (2005). The carboxyl-terminal region of cyclic nucleotide-modulated channels is a gating ring, not a permeation path. Proc. Natl. Acad. Sci. USA 102, 2742-2747.

Kaupp, U.B. and Seifert, R. (2001). Molecular diversity of pacemaker ion channels. Ann. Rev. Physiol. 63, 235-257.

Kaupp, U.B. and Seifert, R. (2002). Cyclic nucleotide-gated ion channels. Physiological reviews $82,769-824$.
Kim, C., Xuong, N.H., and Taylor, S.S. (2005). Crystal structure of a complex between the catalytic and regulatory $(R \mid \alpha)$ subunits of PKA. Science 307, 690-696.

Lolicato, M., Nardini, M., Gazzarrini, S., Moller, S., Bertinetti, D., Herberg, F.W., Bolognesi, M., Martin, H., Fasolini, M., Bertrand, J.A., et al. (2011). Tetramerization dynamics of C-terminal domain underlies isoform-specific CAMP gating in hyperpolarization-activated cyclic nucleotide-gated channels. J. Biol. Chem. 286, 44811-44820.

Mari, S.A., Pessoa, J., Altieri, S., Hensen, U., Thomas, L., Morais-Cabral, J.H., and Muller, D.J. (2011). Gating of the MlotiK1 potassium channel involves large rearrangements of the cyclic nucleotide-binding domains. Proc. Natl. Acad. Sci. USA 108, 20802-20807.

Marques-Carvalho, M.J., Sahoo, N., Muskett, F.W., Vieira-Pires, R.S., Gabant, G., Cadene, M., Schonherr, R., and Morais-Cabral, J.H. (2012). Structural, biochemical, and functional characterization of the cyclic nucleotide binding homology domain from the mouse EAG1 potassium channel. J. Mol. Biol. 423, 34-46.

Matulef, K., Flynn, G.E., and Zagotta, W.N. (1999). Molecular rearrangements in the ligand-binding domain of cyclic nucleotide-gated channels. Neuron 24, 443-452.

McKay, D.B. and Steitz, T.A. (1981). Structure of catabolite gene activator protein at $2.9 \AA$ resolution suggests binding to left-handed B-DNA. Nature 290, 744-749.

Nimigean, C.M., Shane, T., and Miller, C. (2004). A cyclic nucleotide modulated prokaryotic K+ channel. J. Gen. Physiol. 124, 203-210.

Peuker, S., Cukkemane, A., Held, M., Noe, F., Kaupp, U.B., and Seifert, R. (2013). Kinetics of ligand-receptor interaction reveals an induced-fit mode of binding in a cyclic nucleotideactivated protein. Biophys. J. 104, 63-74.

Puljung, M.C. and Zagotta, W.N. (2013). A secondary structural transition in the C-helix promotes gating of cyclic nucleotideregulated ion channels. J. Biol. Chem. 288, 12944-12956.

Rehmann, H., Prakash, B., Wolf, E., Rueppel, A., de Rooij, J., Bos, J.L., and Wittinghofer, A. (2003). Structure and regulation of the cAMP-binding domains of Epac2. Nat. Struct. Biol. 10, 26-32.

Rehmann, H., Das, J., Knipscheer, P., Wittinghofer, A., and Bos, J.L. (2006). Structure of the cyclic-AMP-responsive exchange factor Epac2 in its auto-inhibited state. Nature 439, 625-628.

Rehmann, H., Wittinghofer, A., and Bos, J.L. (2007). Capturing cyclic nucleotides in action: snapshots from crystallographic studies. Nat. Rev. Mol. Cell. Biol. 8, 63-73.

Rehmann, H., Arias-Palomo, E., Hadders, M.A., Schwede, F., Llorca, O., and Bos, J.L. (2008). Structure of Epac2 in complex with a cyclic AMP analogue and RAP1B. Nature 455, 124-127.

Robinson, R.B. and Siegelbaum, S.A. (2003). Hyperpolarizationactivated cation currents: from molecules to physiological function. Ann. Rev. Physiol. 65, 453-480.

Schünke, S., Novak, K., Stoldt, M., Kaupp, U.B., and Willbold, D. (2007). Resonance assignment of the cyclic nucleotide binding domain from a cyclic nucleotide-gated $\mathrm{K}^{+}$channel in complex with cAMP. Biomol. NMR Assign. 1, 179-181.

Schünke, S., Stoldt, M., Novak, K., Kaupp, U.B., and Willbold, D. (2009). Solution structure of the Mesorhizobium loti K1 channel cyclic nucleotide-binding domain in complex with CAMP. EMBO Rep. 10, 729-735.

Schünke, S., Stoldt, M., Lecher, J., Kaupp, U.B., and Willbold, D. (2011). Structural insights into conformational changes 
of a cyclic nucleotide-binding domain in solution from Mesorhizobium loti K1 channel. Proc. Natl. Acad. Sci. USA 108, 6121-6126.

Su, Y., Dostmann, W.R., Herberg, F.W., Durick, K., Xuong, N.H., Ten Eyck, L., Taylor, S.S., and Varughese, K.I. (1995). Regulatory subunit of protein kinase A: structure of deletion mutant with cAMP binding domains. Science 269, 807-813.

Taraska, J.W., Puljung, M.C., Olivier, N.B., Flynn, G.E., and Zagotta, W.N. (2009). Mapping the structure and conformational movements of proteins with transition metal ion FRET. Nat. Methods 6, 532-537.

Tibbs, G.R., Liu, D.T., Leypold, B.G., and Siegelbaum, S.A. (1998). A state-independent interaction between ligand and a conserved arginine residue in cyclic nucleotide-gated channels reveals a functional polarity of the cyclic nucleotide binding site. J. Biol. Chem. 273, 4497-4505.

Vaandrager, A.B. and de Jonge, H.R. (1996). Signalling by cGMP-dependent protein kinases. Mol. Cell. Biol. 157, 23-30.

Varnum, M.D., Black, K.D., and Zagotta, W.N. (1995). Molecular mechanism for ligand discrimination of cyclic nucleotide-gated channels. Neuron 15, 619-625.

Wainger, B.J., DeGennaro, M., Santoro, B., Siegelbaum, S.A., and Tibbs, G.R. (2001). Molecular mechanism of cAMP modulation of HCN pacemaker channels. Nature 411, 805-810.

Wang, J., Chen, S., and Siegelbaum, S.A. (2001). Regulation of hyperpolarization-activated HCN channel gating and CAMP

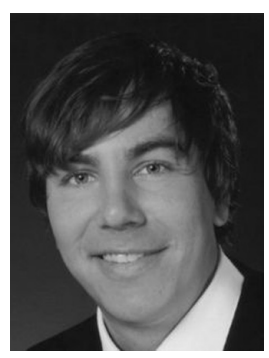

Sven Schünke studied Biology in Düsseldorf, Germany, and obtained his PhD in 2010 under Dieter Willbold at the Institute for Physical Biology at the Heinrich-Heine University Düsseldorf and at the Institute for Structural Biochemistry at the Jülich Research Center on structure analysis of CNBDs using NMR spectroscopy. As a Postdoctoral Research Officer, he is working on the functional and structural characterization of neuronal and viral proteins. modulation due to interactions of $\mathrm{COOH}$ terminus and core transmembrane regions. J. Gen. Physiol. 118, 237-250.

Weber, I.T. and Steitz, T.A. (1987). Structure of a complex of catabolite gene activator protein and cyclic AMP refined at 2.5 A resolution. J. Mol. Biol. 198, 311-326.

Xu, X., Vysotskaya, Z.V., Liu, Q., and Zhou, L. (2010). Structural basis for the cAMP-dependent gating in the human HCN4 channel. J. Biol. Chem. 285, 37082-37091.

Xu, X., Marni, F., Wu, S., Su, Z., Musayev, F., Shrestha, S., Xie, C., Gao, W., Liu, Q., and Zhou, L. (2012). Local and global interpretations of a disease-causing mutation near the ligand entry path in hyperpolarization-activated CAMP-gated channel. Structure 20, 2116-2123.

Zagotta, W.N., Olivier, N.B., Black, K.D., Young, E.C., Olson, R., and Gouaux, E. (2003). Structural basis for modulation and agonist specificity of HCN pacemaker channels. Nature 425, 200-205.

Zhou, L. and Siegelbaum, S.A. (2007). Gating of HCN channels by cyclic nucleotides: residue contacts that underlie ligand binding, selectivity, and efficacy. Structure 15, 655-670.

Zhou, L., Olivier, N.B., Yao, H., Young, E.C., and Siegelbaum, S.A. (2004). A conserved tripeptide in CNG and HCN channels regulates ligand gating by controlling $\mathrm{C}$-terminal oligomerization. Neuron 44, 823-834.

Zubay, G., Schwartz, D., and Beckwith, J. (1970). Mechanism of activation of catabolite-sensitive genes: a positive control system. Proc. Natl. Acad. Sci. USA 66, 104-110.

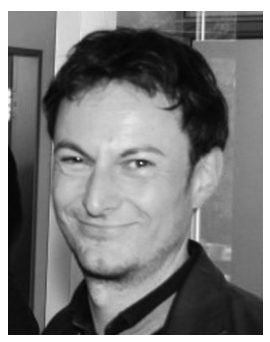

Matthias Stoldt studied Biochemistry in Halle, Germany, and obtained his PhD in 2001 at the Institute for Molecular Biotechnology and at the Friedrich-Schiller University Jena on NMR structure determination of a ribosomal protein-RNA complex. Today he is an Assistant Professor at the Institute for Physical Biology in Düsseldorf, Germany, and a Group Leader for protein NMR spectroscopy at the Institute for Structural Biochemistry at the Jülich Research Center, Germany. 